\title{
Application of the multiplicative regularized contrast source inversion method on TM- and TE-polarized experimental Fresnel data
}

\author{
Aria Abubakar ${ }^{1}$, Peter M van den Berg ${ }^{2}$ and Tarek M Habashy ${ }^{1}$ \\ ${ }^{1}$ Schlumberger-Doll Research, 36 Old Quarry Road, Ridgefield, CT 06877, USA \\ ${ }^{2}$ Faculty of Applied Sciences, Delft University of Technology, 2628 CJ Delft, The Netherlands \\ E-mail: aabubakar@slb.com
}

Received 21 March 2005, in final form 29 May 2005

Published 25 November 2005

Online at stacks.iop.org/IP/21/S5

\begin{abstract}
This paper presents the results of profile inversion of multi-frequency electromagnetic scattered field data, measured by the Institute Fresnel (Marseille, France), from cylindrical objects, both for TM and TE illuminations. The reconstructions are obtained by applying the multiplicative regularized contrast source inversion (MR-CSI) method. Since we are dealing with configurations consisting of both dielectric and metallic objects, we reconstruct both the permittivity and the conductivity of the unknown objects. The results show that the MR-CSI method successfully performs 'blind' inversion of a wide class of scattered field data.
\end{abstract}

(Some figures in this article are in colour only in the electronic version)

\section{Introduction}

We discuss the performance of the contrast source inversion (CSI) method [1, 2], enhanced with a multiplicative regularization technique (MR-CSI) [3]. Although the CSI method was already effective for the inversion of the experimental Ipswich data [4], its updated version, the MR-CSI method, shows a significant improvement in inverting the first set of data measured by the Institut Fresnel (Marseille, France) [5]. The inversion results obtained using the MR-CSI method from these first Fresnel data sets were presented in [6]. Following these experiments, the MR-CSI method has been improved by the introduction of the so-called weighted $L_{2}$-norm regularizer, see [7]. The inversion results of the first Fresnel data set using the MR-CSI method with weighted $L_{2}$-norm regularizer can be found in [8].

With this version of the MR-CSI method, we demonstrate the reconstructions from the second set of data measured by the Institut Fresnel. We carry out a 'blind' inversion of these data sets without explicitly taking into consideration any a priori information regarding the type 
of objects (either dielectric or metallic) to be reconstructed. In all cases, we reconstruct both the permittivity and the conductivity of the unknown objects. The only a priori information which is used in the inversion is the positivity constraint on both permittivity and conductivity. The inversion results show that the MR-CSI method seems to handle the experimental field data very well.

\section{Definitions}

The Institute Fresnel experimental setup consists of a transmitting and a receiving antenna, both of which are double-ridged horn antennas. The antennas are moved on a circular rail around the object(s). The objects are elongated in the direction perpendicular to the plane in which the antennas are rotated (i.e., the plane of measurement), so that a two-dimensional (2D) model is appropriate. In the plane of illumination, we choose a $2 \mathrm{D}$ rectangular test domain $D$ containing the object(s). The transmitting antenna illuminates the objects from different locations distributed equidistantly around the object. We use the subscript $j$ to denote the measured frequency and the subscript $s$ to denote the dependence on the transmitter position. The receiving antenna measures the total field and the incident field from a number of different locations distributed equidistantly around the object. The scattered field, which is needed in the inversion, can then be found by subtracting the incident field from the total field.

The experimental data are collected at a number of frequencies with time factor $\exp \left(-\mathrm{i} \omega_{j} t\right)$, where $\mathrm{i}^{2}=-1, \omega_{j}$ is the radial frequency and $t$ is the time. We introduce the vectors $\boldsymbol{p}$ and $\boldsymbol{q}$ as the spatial positions in 2D. We use the Maxwell model for the constitutive parameters of the object. Hence, the contrast function for each frequency is defined as follows:

$$
\chi_{j}(\boldsymbol{q})=\frac{\varepsilon(\boldsymbol{q})-\varepsilon_{0}}{\varepsilon_{0}}+\mathrm{i} \frac{\sigma(\boldsymbol{q})}{\omega_{j} \varepsilon_{0}},
$$

where $\varepsilon$ and $\sigma$ denote the permittivity and conductivity, which are frequency independent. The symbol $\varepsilon_{0}$ denotes the permittivity in vacuum. Since $\varepsilon$ and $\sigma$ are frequency independent, it is obvious that in the inversion we need only to invert for one value of the contrast function. Let $\chi_{1}$ be the contrast function value at the angular frequency $\omega_{1}$, then the other values of the contrast as a function of frequency can be calculated through

$$
\chi_{j}(\boldsymbol{q})=\operatorname{Re}\left[\chi_{1}(\boldsymbol{q})\right]+\mathrm{i} \frac{\omega_{1}}{\omega_{j}} \operatorname{Im}\left[\chi_{1}(\boldsymbol{q})\right] .
$$

Since all the objects lie inside a test domain $D$, the contrast function is therefore non-zero inside $D$ and zero elsewhere.

In the TM case where the non-zero component of the electric field is the only one parallel to the cylindrical objects, we deal with a scalar wave field problem. The domain integral representation for the scattered field as a function of the total field $u_{s, j}$ and the contrast $\chi_{j}$ is given by

$u_{s, j}^{\mathrm{sct}}(\boldsymbol{p})=K_{j}^{\mathrm{TM}}\left[\chi_{j} u_{s, j}\right]=k_{0, j}^{2} \int_{D} g_{j}(\boldsymbol{p}, \boldsymbol{q}) \chi_{j}(\boldsymbol{q}) u_{s, j}(\boldsymbol{q}) \operatorname{dv}(\boldsymbol{q}), \quad \boldsymbol{p} \in S$,

where $k_{0, j}=\omega_{j} \sqrt{\varepsilon_{0} \mu_{0}}$ is the wave number in free space and $S$ is the data domain where the transmitter and receiver are located. The scalar homogeneous Green function is given by

$$
g_{j}(\boldsymbol{p}, \boldsymbol{q})=\frac{\mathrm{i}}{4} H_{0}^{(1)}\left(k_{0, j}|\boldsymbol{p}-\boldsymbol{q}|\right),
$$

where $H_{0}^{(1)}$ denotes the first kind Hankel function of zero order.

In the TE case, the field quantities are two-component vectors representing the electric field components in the transversal plane of the cylindrical objects. The domain integral 
representation for the scattered field vector as a function of the total field $\boldsymbol{u}_{s, j}$ and the contrast $\chi_{j}$ is given by

$\boldsymbol{u}_{s, j}^{\mathrm{sct}}(\boldsymbol{p})=K_{j}^{\mathrm{TE}}\left[\chi_{j} \boldsymbol{u}_{s, j}\right]=\left(k_{0, j}^{2}+\nabla \nabla\right) \int_{D} g_{j}(\boldsymbol{p}, \boldsymbol{q}) \chi_{j}(\boldsymbol{q}) \boldsymbol{u}_{s, j}(\boldsymbol{q}) \operatorname{dv}(\boldsymbol{q}), \quad \boldsymbol{p} \in S$,

where $\nabla$ is the spatial differentiation operator with respect to $\boldsymbol{p}$.

We use the symbol $u_{s, j}$ for both the scalar field $u_{s, j}$ in the TM case and the vector field $\boldsymbol{u}_{s, j}$ in the TE case. Hence, the total field, $u_{s, j}=u_{s, j}^{\mathrm{inc}}+u_{s, j}^{\mathrm{sct}}$, and the contrast inside the test domain $D$ satisfy the following integral equation:

$$
u_{s, j}^{\text {inc }}(\boldsymbol{p})=u_{s, j}(\boldsymbol{p})-K_{j}^{\{\mathrm{TM}, \mathrm{TE}\}}\left[\chi_{j, n} u_{s, j, n}\right], \quad \boldsymbol{p} \in D
$$

where the operators $K_{j}^{\mathrm{TM}}\left[\chi_{j} \boldsymbol{u}_{s, j}\right]$ and $K_{j}^{\mathrm{TE}}\left[\chi_{j} \boldsymbol{u}_{s, j}\right]$ are defined in (3) and (5), for the TM case and TE case, respectively. Equations (3), (5) and (6) are the basic equations for developing any inversion algorithm based on the integral equation formulation. The goal of solving the inverse scattering problem can be formulated as follows: solve (3) or (5) to obtain the contrast $\chi_{1}$ on $D$ from the knowledge of the scattered field $u_{s, j}^{\text {sct }}$ on $S$ and the incident field $u_{s, j}^{\text {inc }}$ on $D$ subject to the necessary condition that the total field $u_{s, j}$ on $D$ and the contrast $\chi_{1}$ on $D$ satisfy the integral equation in (6).

We consider the inverse scattering problem as an optimization problem where, in each iteration $n$, we update the contrast sources $w_{s, j, n}$ and the contrast $\chi_{j, n}$ alternatingly, by minimization of the cost functional

$F_{n}=\left\{\frac{\sum_{j} \sum_{s}\left\|u_{s, j}^{\mathrm{sct}}-K_{j}^{\{\mathrm{TM}, \mathrm{TE}\}}\left[w_{s, j, n}\right]\right\|_{S}^{2}}{\sum_{j} \sum_{s}\left\|u_{s, j}^{\mathrm{sct}}\right\|_{S}^{2}}+\frac{\sum_{j} \sum_{s}\left\|w_{s, j, n}-\chi_{j, n} u_{s, j, n}\right\|_{D}^{2}}{\sum_{j} \sum_{s}\left\|\chi_{j, n-1} u_{s, j}^{\mathrm{inc}}\right\|_{D}^{2}}\right\}$,

where

$$
u_{s, j, n}=u_{s, j}^{\text {inc }}+K_{j}^{\{\mathrm{TM}, \mathrm{TE}\}}\left[w_{s, j, n}\right],
$$

and $\|\cdot\|_{S}^{2}$ and $\|\cdot\|_{D}^{2}$ denote the $L_{2}$-norm on the data domain $S$ and the object domain $D$, respectively. In this CSI method, we use the back-propagation step to arrive at initial estimates for the contrast sources and the contrast. We further include a multiplicative regularization. Details of this so-called MR-CSI method for the multi-frequency problem can be found in [6]. However, the procedure to update the contrast function is replaced by the improved version in [8]. The optimization process may be terminated if one of the following stopping conditions is satisfied:

- The difference between the normalized data error $F_{n}$ at two successive iterates, $n$th and $(n-1)$ th, is within a prescribed error quantity (it is set to be $10^{-5}$ ).

- The total number of iterations exceeds a prescribed maximum $N_{\max }=1024$.

The a priori information that the permittivity and the conductivity are positive is implemented by enforcing the negative value to zero after each iteration. This simple procedure is employed in all of the inversion runs.

\section{Reconstruction results}

In the experimental setup, the fields are generated and received by horn antennas. However, as we previously argued, the problem is predominantly 2D. Hence, both receivers and transmitters are approximated as line receivers and line transmitters. Therefore, we carry out the calibration procedure outlined in [6]. 

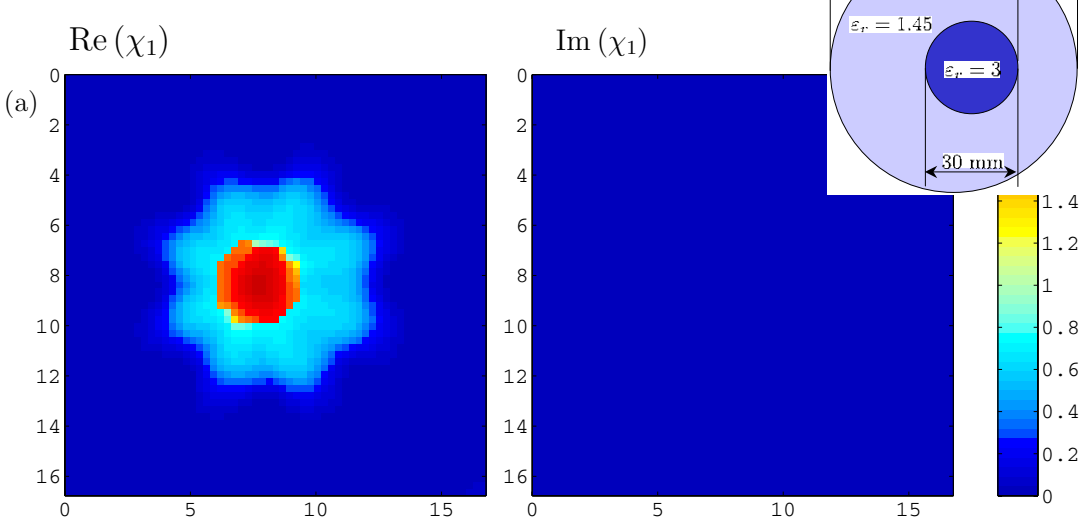

(b)
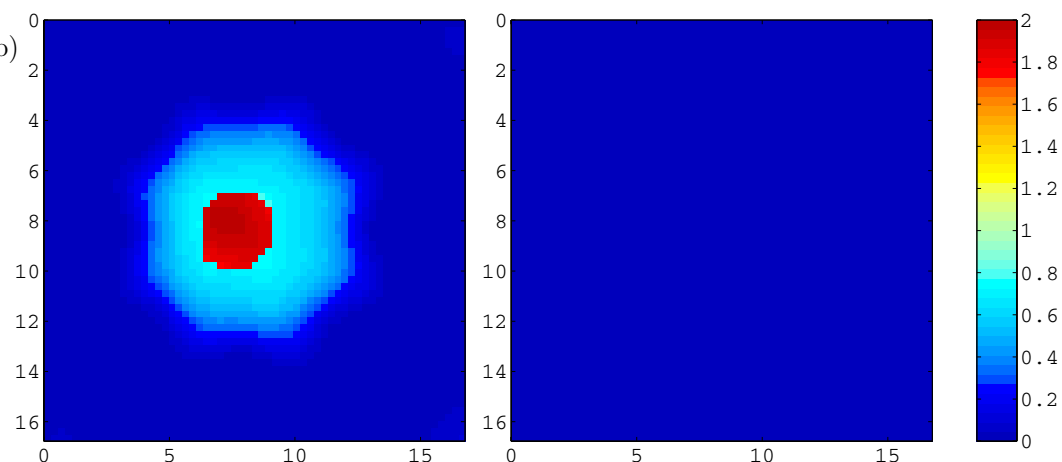

Figure 1. Reconstruction of the configuration with a dielectric cylinder embedded in another dielectric cylinder both for TM polarization (data set: FoamDiellntTM) (a) and TE polarization (data set: FoamDiellntTE) (b)

In the inversion, we take a test domain $D$ of $16.775 \mathrm{~cm} \times 16.775 \mathrm{~cm}$. Unless stated otherwise, this test domain $D$ is discretized into $61 \times 61$ rectangular subdomains. The side length of each subdomain is $0.275 \mathrm{~cm}$.

The data for different frequencies are inverted simultaneously. In the figures, we plot the complex contrast function $\chi_{1}$ only. This is the complex contrast at the lowest frequency.

For the different configurations measured, we have obtained the following reconstructions.

\subsection{Data FoamDiellntTM and FoamDiellntTE}

We first consider the configuration with a circular dielectric cylinder with a diameter of $30 \mathrm{~mm}$ embedded in another circular dielectric cylinder with a diameter of $80 \mathrm{~mm}$. The smaller cylinder has a relative permittivity value of $\varepsilon_{\mathrm{r}}=3$ while the larger cylinder has a relative permittivity value of $\varepsilon_{\mathrm{r}}=1.45$. Both cylinders are non-conductive. The schematic of these cylinders are shown in the top-right side of figure 1 . In the experiment, there are eight transmitters distributed uniformly on a circle with a radius of $1.67 \mathrm{~m}$ from the centre of the 

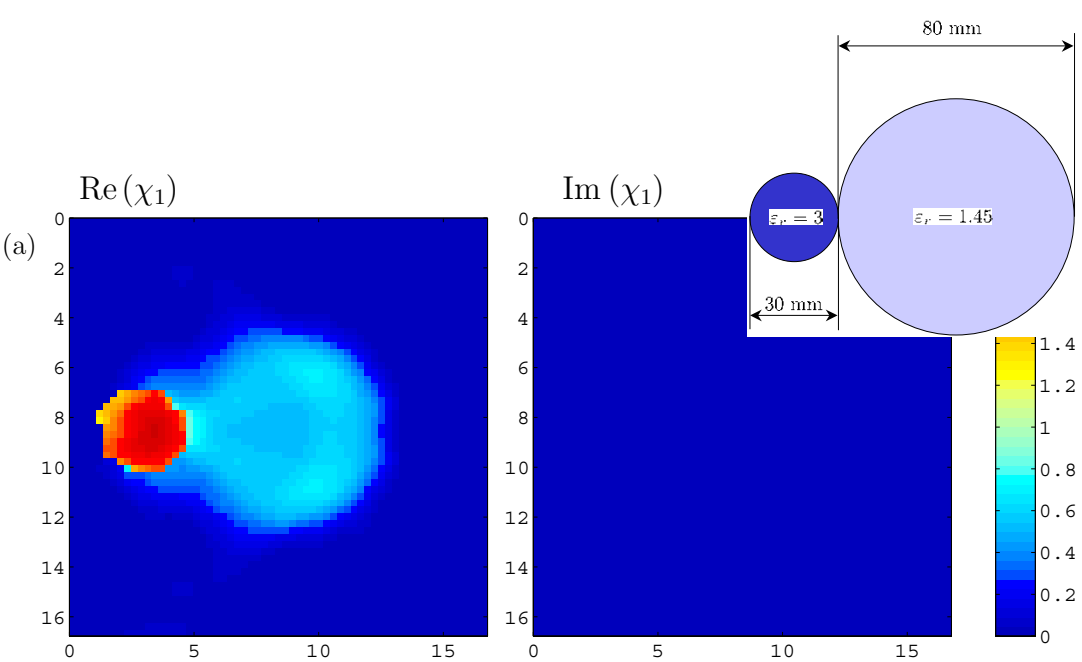

(b)
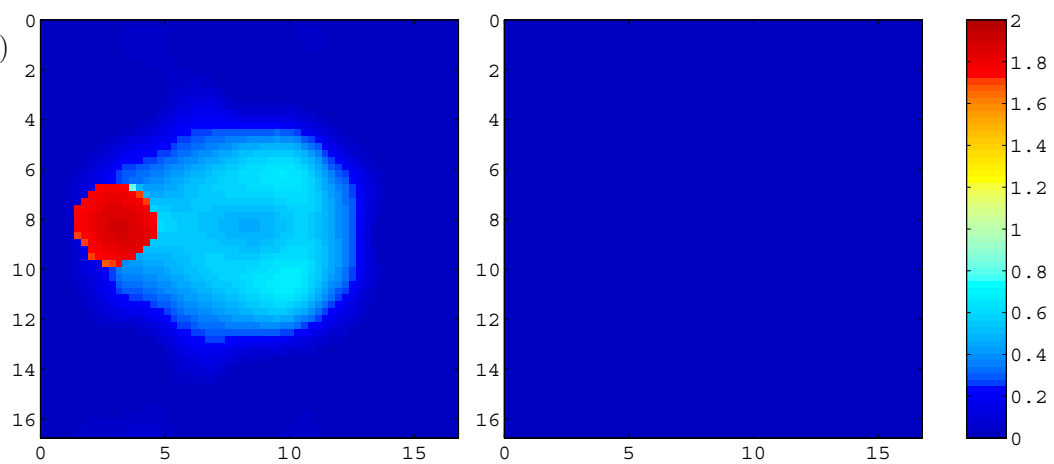

Figure 2. Reconstruction of the configuration with two disjoint dielectric cylinders both for TM polarization (data set: FoamDielExtTM) (a) and TE polarization (data set: FoamDielExtTE) (b).

experimental setup. For each transmitter, the data are measured using 241 receivers located on a circle with a radius of $1.67 \mathrm{~m}$. The data are gathered at nine frequencies in the range of 2-10 GHz. The wavelength at $10 \mathrm{GHz}$ is equal to $3 \mathrm{~cm}$, so the dimensions of the test domain $D$ are equal to $5.5 \times 5.5$ wavelength. All the data at nine frequencies are simultaneously inverted. In the inversion, we have used the a priori knowledge that neither the permittivity nor the conductivity can be negative.

The real part (the left plots) and the imaginary part (the right plots) of the reconstructed contrast $\chi_{1}$ are presented in figure 1. Both reconstructions from the TM data set (the top plots) and TE data set (the bottom plots) show that the relative permittivity values, $\varepsilon_{\mathrm{r}}=\operatorname{Re}\left(\chi_{1}\right)+1$, are very well reconstructed. The relative permittivities of the reconstructed small cylinder are around 2.8 (from the TM data) and 2.95 (from the TE data). The vanishing imaginary part of the contrast function indicates that the objects are purely dielectric (non-conductive).

\subsection{Data FoamDielExtTM and FoamDielExtTE}

In the second configuration, we consider the case that the small internal circular cylinder is now located outside the larger circular cylinder. The data are collected in the same way 

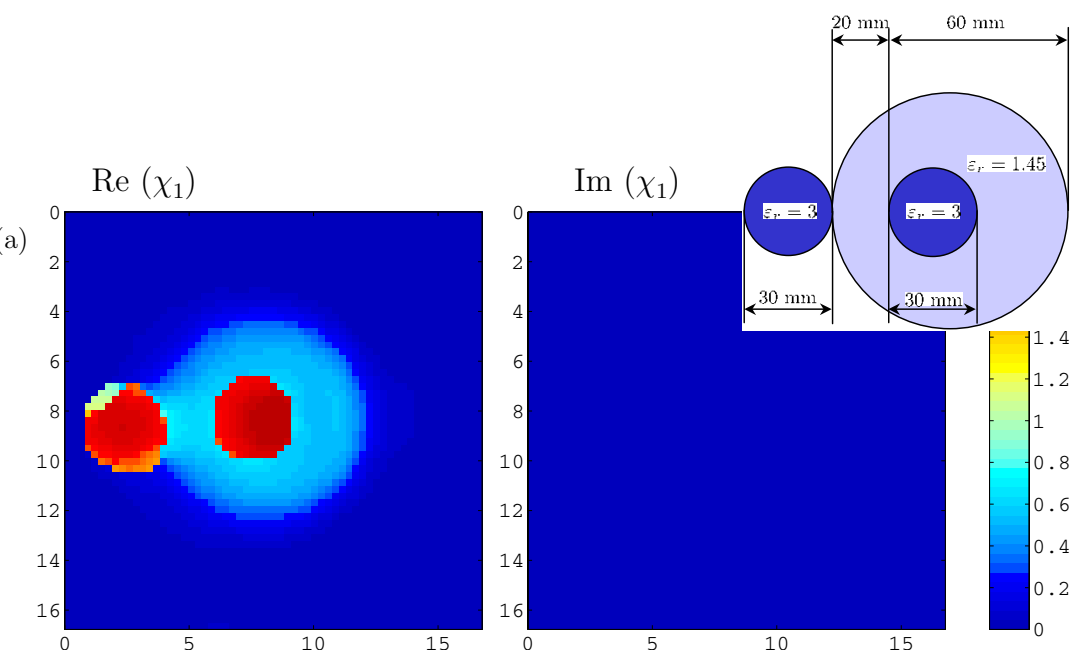

(b)
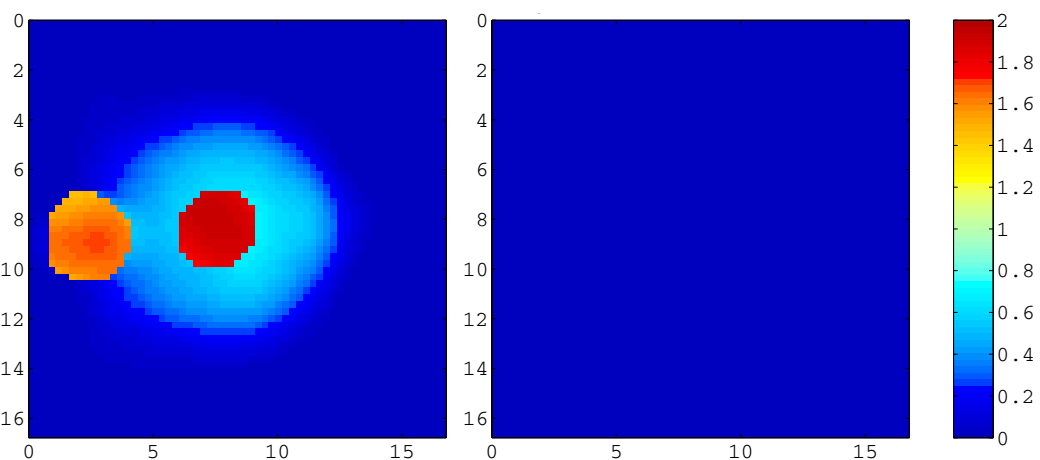

Figure 3. Reconstruction of the configuration with a dielectric cylinder embedded in another dielectric cylinder and a third disjoint dielectric cylinder both for TM polarization (data set: FoamTwinDielTM) (a) and TE polarization (data set: FoamTwinDielTE) (b).

as the previous experiment. The reconstructed images are presented in figure 2 . We observe that for the present configuration the larger cylinder is better reconstructed from the TM data set.

\subsection{Data FoamTwinDielTM and FoamTwinDielTE}

The third configuration is the combination of both previous configurations. The two smaller circular dielectric cylinders have relative permittivity values of $\varepsilon_{\mathrm{r}}=3$ while the larger circular dielectric cylinder has a relative permittivity value of $\varepsilon_{\mathrm{r}}=1.45$. In contrast to the previous two experiments, the data are obtained using 18 transmitters. The numbers of frequencies and receivers remain the same.

The inversion is carried out in a similar way as in the previous experiments. The reconstructed images presented in figure 3 show that the qualitative results are very similar to the ones of figure 1. However, we note also that the circular form of the larger dielectric cylinder is better reconstructed; this is caused by the additional numbers of transmitter used in the inversion. 
(a) $\operatorname{Re}\left(\chi_{1}\right)$

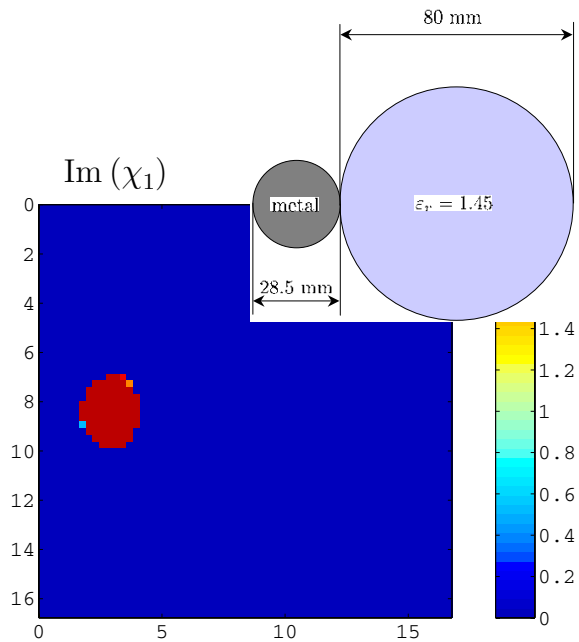

(b)
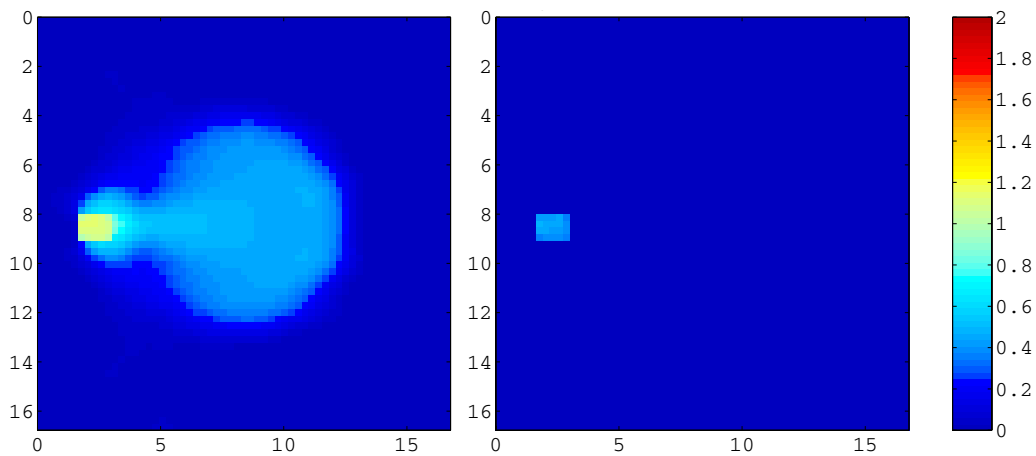

Figure 4. Reconstruction of the configuration with two disjoint cylinders, a dielectric one and a metallic one, both for TM polarization (data set: FoamMetExtTM) (a) and TE polarization (data set: FoamMetExtTE) (b); the test domain $D$ is subdivided into $61 \times 61$ subdomains.

\subsection{Data FoamMetExtTM and FoamMetExtTE}

The fourth configuration consists of one circular dielectric cylinder with a relative permittivity value of $\varepsilon_{\mathrm{r}}=1.45$ and one metallic cylinder with a diameter of $28.5 \mathrm{~mm}$. The data are now collected at 17 frequencies in the range of $2-18 \mathrm{GHz}$. The wavelength at $18 \mathrm{GHz}$ is $1.67 \mathrm{~cm}$; hence, the width and height of the test domain $D$ are ten times the wavelength. The number of transmitters is 18 while the number of receivers is 241 . All frequencies are simultaneously used in the inversion procedure. In both TM and TE cases, we enforce positivity in the permittivity and in the conductivity.

The reconstructed images from the TM and TE data sets are shown in figures 4(a) and (b). For the frequency of operation of $18 \mathrm{GHz}$, the discretization mesh of the test domain $D$ may be too coarse. Within the present discretization, the mesh size is approximately equal to six points per wavelength. We therefore refine our mesh from $61 \times 61$ subdomains into $122 \times$ 122 subdomains and rerun our inversion code. The reconstructed images are now shown in figure 5.

The inversion results from the TM data set are presented in figure 5(a). This figure shows that the metallic cylinder is retrieved with real and imaginary parts having the same order of magnitude. These inversion results also show that there is an ambiguity in the inversion. In 

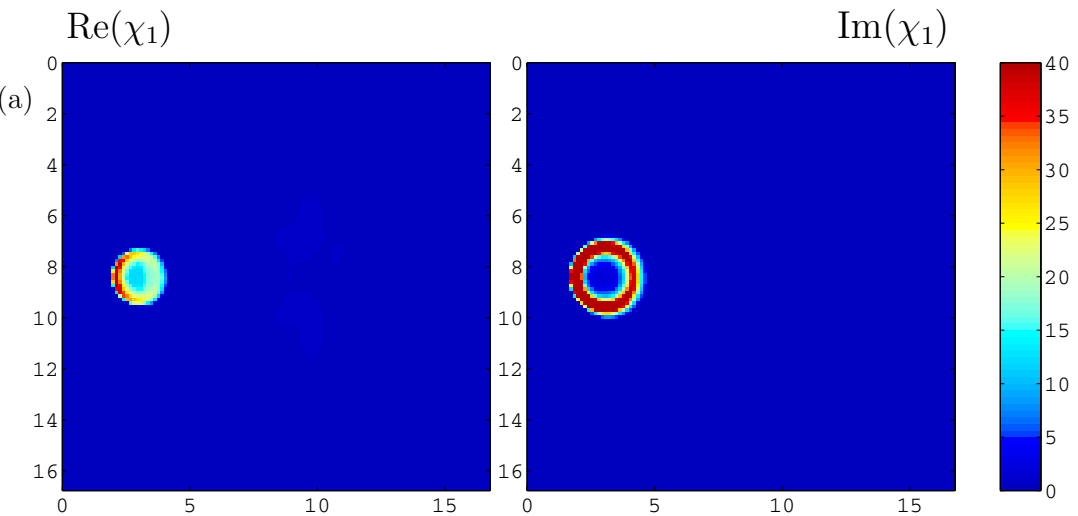

(b)
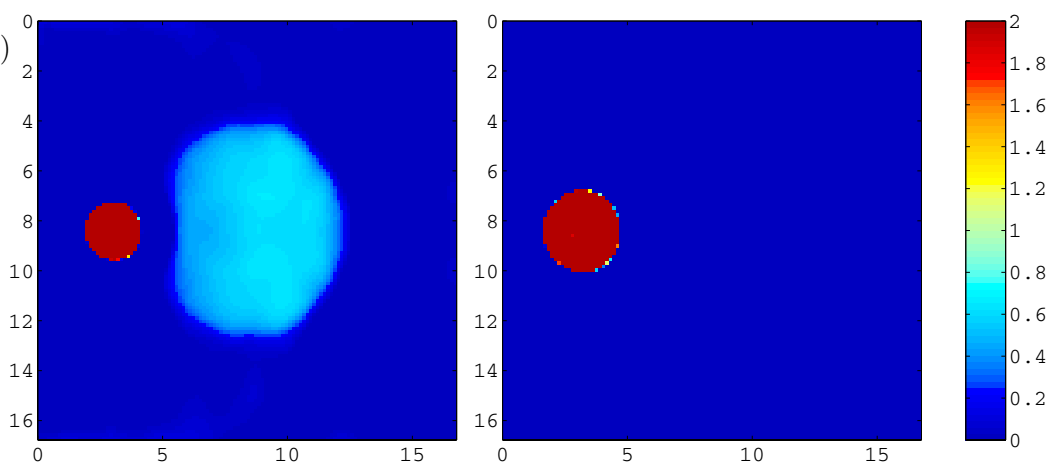

Figure 5. Reconstruction of the configuration with two disjoint cylinders, a dielectric one and a metallic one, for TM polarization using $122 \times 122$ subdomains, for plot scales of $0-40$ (a) and 0-2 (b).
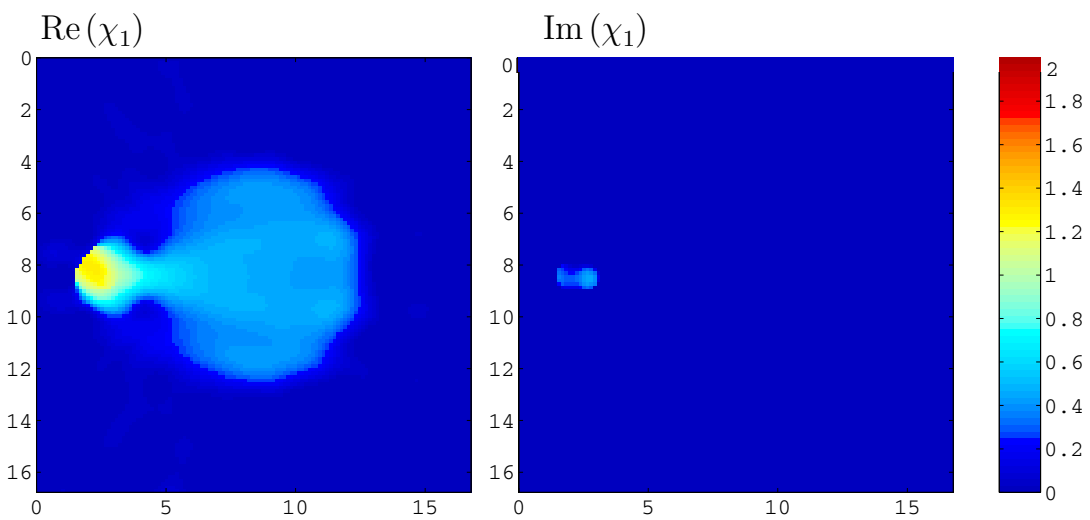

Figure 6. Reconstruction of the configuration with two disjoint cylinders, a dielectric one and a metallic one, for TE polarization using $122 \times 122$ subdomains.

principle, when carrying out the inversion of a perfectly conducting cylinder, one can only reconstruct uniquely the boundary of the object. Inside the metal object, the contrast sources are invisible, with the consequence that any contrast inside the object may be arbitrarily arrived at. The small circular object with a large permittivity value appearing in the image of $\operatorname{Re}\left(\chi_{1}\right)$ is obviously an artefact of the inversion algorithm. However, since the reconstructed 
circular object in $\operatorname{Re}\left(\chi_{1}\right)$ lies completely inside the circular cylinder $\operatorname{in} \operatorname{Im}\left(\chi_{1}\right)$ with a very high amplitude $(\approx 40)$, one can conclude that we are dealing with a metallic object. This artefact can only be eliminated by enforcing the a priori information that the object is metallic. One way to enforce this constraint is to force the real part of the contrast function to zero at spatial locations where the imaginary part of the contrast surpasses a certain large value. We plot again the reconstructed $\chi_{1}$ in figure 5(b), however, this time we plotted the contrast only up to a maximum value of 2 . From this figure we observe that the algorithm reconstructs very well the shape and the permittivity of the circular dielectric object.

On the other hand, the imaginary parts of the contrast of the TE inversion do not exhibit any significant features (see figure 6). It seems that only a real part of the contrast is reconstructed. A similar observation can be made from the reconstruction results of the rectangular metallic cylinder of the first Fresnel data set, see [6]. We surmise that this false reconstruction is due to the fact that we measure only one electric field component, namely, the electric field component perpendicular to the radial direction, while inside the test domain $D$ we deal with a vectorial problem of two unknown electric field vectors.

\section{Conclusions}

In view of the present results and our crude approximation of the transmitting and receiving antennas, the multiplicative regularized contrast source inversion method seems to be very robust and is capable of 'blindly' handling a wide class of inverse scattering problems.

Finally, we note that we also can invert both TM and TE data simultaneously. However, since the optimal choice of the weighting factors of the two data sets is not trivial and needs further research, we refrain ourselves in presenting these joint inversion results.

\section{Acknowledgments}

The authors wish to thank Dr M Saillard and Dr K Belkebir for providing their second set of experimental data as an objective test of our inversion algorithm.

\section{References}

[1] Habashy T M, Oristaglio M L and De Hoop A T 1994 Simultaneous nonlinear reconstruction of two-dimensional permittivity and conductivity Radio Sci. 29 1101-18

[2] van den Berg P M and Kleinman R E 1997 A contrast source inversion method Inverse Problems 13 1607-20

[3] van den Berg P M, van Broekhoven A L and Abubakar A 1999 Extended contrast source inversion Inverse Problems 15 1325-44

[4] van den Berg P M, Kooij B J and Kleinman R E 1997 Image reconstruction from Ipswich data: II IEEE Antennas Propag. Mag. 39 29-32

[5] Belkebir K and Saillard M 2001 Guest Editors' introduction. Special section: Testing inversion algorithms against experimental data Inverse Problems 17 1565-71

[6] Bloemenkamp R F, Abubakar A and van den Berg P M 2001 Inversion of experimental multi-frequency data using the contrast source inversion method Inverse Problems 17 1611-22

[7] Abubakar A, van den Berg P M and Mallorqui J J 2002 Imaging of biomedical data using a multiplicative regularized contrast source inversion method IEEE Trans. Microw. Theory Tech. 50 1761-71

[8] van den Berg P M, Abubakar A and Fokkema J T 2003 Multiplicative regularization for contrast profile inversion Radio Sci. 38 23.1-10 